bahnen beschränkt war - bleibt eine offene Frage. Angenommen könnte immerhin werden, dass die rascb verlaufende rareficirende 0 stitis bei ebenfalls acut auftretender interstitieller Nephritis die zunächst liegenden $\mathrm{CO}^{2}$-armen arteriellen Blutbahnen zur Ablage wählte.

Bevor ich schliesse, möclte ich der physiologischen Ablagerung von Kalksalzen in der Knochensubstanz erwähoen. Gestüfzt darauf, dass bei der Verkalkung der Knorpel die Kalkinfiltration als Grenze des Gefässgebietes auftritt, wo keine Lymphgefăsse die überschüssigen Ernährungsflüssigkeiten abführen, erklärt sich Rindfleisch das Ausfallen der Kalksalze in der Weise, dass „das Lösungsmittel derselben, die freie $\mathrm{CO}^{2}$, bei ihrem grossen Diffusionsvermögen die stagnirende $\mathbf{E r}$ nährungsflüssigkeit verlässt - auf anderen Wegen zur Ausscheidung gelangt, bei jhrem Entweichen aber die Kalksalze ungelöst zurücklässt.“ Wäre es nicht möglich, die Kalkmetastase der Lungen in äbnlicher Weise zu erklären: die Lungenalveolen sind die Grenzen verschiedenen koblensãurehaltigen Blutes, - die ausgeathmete freie $\mathrm{CO}^{2}$ suspendirt die Kalksalze, so sie überschüssig sind, und überlässt sie dem an freier $\mathrm{CO}^{2}$ relativ armen arteriellen Blute und den Eraährungsflüssigkeiten.

4.

\title{
Ueber einige Fälle von Heilung des Prolapsus uteri ohne mechanische Mittel.
}

\author{
Von Nicolai Andreeff, Stud. med. in Kasan.
}

Ich habe bemerkt, dass eine alkoholische Jodlösung; bei einigen Fällen von Uterusleiden angewandt, so auf die Bänderapparate wirkt, dass sie ans dem erschlafften und geschwächten Zustande in einen mehr normalen übergeführt werden.

In Folge dieser Bemerkung entschloss ich mich, die Einwirkung dieser Jodlösung bei vollständigem Descensus und Prolapsus uteri zu versuchen.

Die ersten Versuche gaben mir so gïnstige Resultate, dass ich mich veranlasst fühlte, dieses Mittel nachher in mehreren Fällen systematisch anzuwenden; ich erzielte jedesmal einen sehr günstigen Erfolg und entschloss mich deshalb, meine Erfahrungen den deutschen klinischen Aerzten mitzutheilen und sie ihrer geneigten Aufmerksamkeit zu empfehlen.

Im August 1871 wurde ich mit einer Kranken bekannt, die an einen Prolapsus uteri completus seit 4 Jahren litt. Sie hatte Erlösung von ihrem Leiden in vielen Heilmethoden durch mechanische Mittel gesucht, jedoch, wie dies so oft geschieht, ohne auch nur Erleichterung zu finden.

Ich schlug der Kranken vor, meine Methode za versuchen; die Kranke ging darauf ein und nach einer vierwöchentlichen Behandlung war sie ganz bergestellt. Es war dies eine junge, 22jährige Frau, abgemagert und von schwachem Körperbau, die vor 4 Jahren, bald nach einer Niederkunft erst von einem Descensus und sodann von einem Prolapsus uteri completus befallen wurde. Der Vorfall der 
Gebärmutter war so zur Gewobnheit geworlen, dass es, nach einer Reposition derselben, tur eines starken Fustens der Kranken bedarfte, um die Gebärmotter zwischen den Lenden in beinahe ihrer ganzen Grösse erscheinen zu sehen.

Meine Behandlung bestand in Folgendem :

Die Gebarmutter wurde bei horizontaler Lage der Kranken reponirt; darnach worde mit Huile des Mutterspiegels das Gewölbe der Yagina (d. h. der Theil derselben, der um den Wuttermund herum lhegt) mit $\frac{1}{2}$ Drachme einer alkoholisehen Jod̆bsug, die aus einem Theile (nach Vol) Tinctura jodi und einem Theile Alcoholis rectiffcati bestand, bestrichen. Ich verdünote die officinele Tinctura jodi, weil die unverdünhte Tinctur mitunter einen acuten Katnrrh der Vagina und alyst des Vterus herworruft, wis ich dies vorber bei einer fauturin, die an derselben Krankheit litin Gelegenheit hatte zu beobachten. Diese Complication rerging jedoch und die arspringlietre Krankheit ebenfalls.

Nach dem Bestreichen des Gewölbes der Vagina mit Jadjösung blieb die Kranke in einer bequenen horizontalen Lage wa erbielt and taglich Vaginal-Doucben aus reinem Bramenwasser ron $20^{\circ} \mathrm{R}$.

Dieselbe Behandlung mil Jodlösung wurde nach jo 3 Tagen wiederholt und aach einer viermaligen Behandlung, wobei die Stärse der Jodlösung jedesmal erböh wurde, fühlte die hranke silch hergestellt and beendete die Car.

Die Gebarmutter dieser franken senkte sich in Laufe ron 4 Monaten gar nicht mebr'; jetzt ist sie schwanger und ganz gesund.

Nach diesen beiden Fällen von Prolapsus ateri completis gelang es mir, durch dieselbe Methode zwei Fälle von Prolapsus uteri incompletus und fünf Fälle von chronischem Degceasus uteri campletus vollständig za hailes.

Ich wage es oicht, den Leser mit den Krankangeschichten aller dieser Fälle zu ermüden; ich werde mich darauf beschränken, nur einen dieser Fäle, ebenfalls einen Fall por Prolapsus ateri completus zu beschreiben, der in der gynäkologischen Abtheilung des biesigen Stadthospitals, das unter der Leitung meines verehrten Lehrers, des. Hro. Prof. Kosloff steht, rorkam, Die Eohandlung dieses Falles wurde mir von Hrn; Prof. Kosloff gütigst überiassen.

Die Kragke wurde unter No. 1690 in das Aufnahmejournal des Hospitals eingetragen. Warta Gavrilowa, eine Soldatenfran, 50 Jibre alt, won mitelstarkem Körperban, trat in dib gonglologische Abtheilung den 20. September v. J. ein. Sie klagte tiber vollständigen Vorfall der Gebärmutter, det sich vor 5 Monaten gebildet hatte; ubrigens hatte sie schon seit libger Zeit an Deguensus uteri geliten. Die Kranke hatte gur ein Kind, ror 30 Jahren geboren; vor 29 Jabren, in 7. Monate einer neven Schwangerschaft, abortirte sie in Folge ton Hehng von schweren Lasten.

Die Menstruation bat bei the gchon wor 10 Jabren aufgehort,

Die. Untersuchung der Kranlen ergab Folgendes:

Zwiscberi den Lenden bängt ein törper ven ovaler Form, der aus den äusseren Genitalien herworragt. Sein breiteres Ende ist oben bei der Rima pudendi, das schmälere unten. Die Lầnge dieses Körpers betrug ungefăhr 21 Zoli (engl.), die grösste Broite $1 \frac{a}{4}$ Zoll, die Kleinste am freien Ende 1 Zoll. An diesem freien schmalen Ende betindel: sich ebe Querspalte, ron anfgesshwollenen rotben Rändern 
umgeben, worauf sich Erosionen befinden. In die Querspalte konnte das Ende des Zeigefingers eingefübrt werden. Der ganze Körper hatte eine blass-rosenrothe Farbe; er war trocken und auf seiner Oberfläche waren Falten zu sehen, die der äusseren Oeffnung der Genitalien parallel liefen; diese Falten verschwanden am engen Ende des Körpers. Sie hatten das Ansehen, als ob sie einem Sacke angehörten, in welchem sich der herausgefallene Körper befand, da sie sich frei über den Körper, der ziemlich fest war, bewegten. Das Ende des Körpers, wo sich die Querspalte befand, war etwas nach hinten geneigt. Der Börper konote durch leichtes Zusammendrücken in das Becken eingefübrt werden. Es war die berausgefallene Gebärmutter. Die Kranke sagte, dass sie die Gebärmutter gewöhnlich selbst zurückbringe, aber ein paar Schritte führten einen neuen Vorfall derselben herbei.

Da beinahe gar kein Leiden der Vagina vorhanden war, so wurde der Uterus reponirt, und dann das Gewölbe der Vagina mittelst des Mutterspiegels und eines Pinsels, bei horizontaler Lage der Kranken, mit der alkoholischen Jodlösung bestrichen; die Lösung war auch in diesem Falle eine mit Alcohol rectificatus zur Hălfte verdünnte Tinctúra jodi, Eine balbe Stunde spăter bekam die Kranke eine Vaginal-Douche aus Wasser von $18^{\circ} \mathrm{R}$, diese Douche wurde viermal täglich wiederholt. Diese Douchen wurden gegeben, weil ohne dieselben bei vielen Kranken Hitze und Schmerz in der Vagina eintreten, die mitunter einen ziemlich hohen Grad erreichten. Die Quantität der gebrauchten Jodlösung erreichte bei der ersten Bestreichung $\frac{1}{2}$ Drachme. Eine grössere Quantität, besonders bei der ersten Bestreichung, ruft leicht einen Reiz hervor. Die Kranke verblieb in einer bequemen horizontalen Lage und brauchte die Vaginal-Douchen, wie schon gesagt, 3 Tage lang. Zwölf Stunden nach der ersten Bestreichung stand der Uterus beinahe $\frac{1}{2}$ Zoll unter der normalen Stellung. Die Vagina war trocken, etwas hart und erhitzt. Absonderungen aus dem Lterus waren keinerlei Art zu bemerken.

23. September. Die Vagina ist feuchter; der Uterus steht ebenso wie nach der ersten Behandlung; die Temperatur der Vagina ist gefallen, die Vagina ist aber dennoch trockener und wärmer als im normalen Zustande.

26. September. Der Uterus senkt sich nicht, wenn die Kranke eine verticale Stellung annimmt. Die Vagina ist schlaff und weich. Es zeigen sich keine fliessenden Absonderungen aus der Vagina. Es wird zar zweiten Bestreichung mit Jodlösung geschritten, wobei die Mischung ron Tinctura jodi und Alkohol so genommen wurde, dass auf $\frac{2}{3}$ (aach Vol.) Tinctura jodi $\frac{1}{3}$ Alcobolis rectificati kam. Die Quantität der zur Bestreichung gebranchten Flüssigkeit belief sich anf 1 Drachme. Zwölf Stunden nach der zweiten Bestreichung wurde der Uterus an seinem normalen Orte gefunden. In der Vagina dieselben Erscheinungen wie nach der ersten Bestreichung, nur viel schwächer. Die Kranke braucht jetzt Vaginal-Douchen aus Wasser von $20^{\circ}$ R.; aber nur einmal täglich.

4. Detober, Es haben sich Schmerzen in regione scrobiculi cordis and in beiden Hypochondrien eingestellt, ebenso wie ein heftiger Husten. Gegen diese Erscheinungen werden die gewöhnlichen Mittel angewandt.

9. October, Der Uterus steht an seinem normalen Orte. Die Vagina sondert nur etwas mehr Schleim als gewöhnlich ab, zeigt aber im Uebrigen keine anomalen Erscheinungen. 
15. October. Die Lage des Uterus ist dieselbe geblieben. An diesem Tage hört die Kranke anf, kalte Douchen zu brauchen.

27. Octoher, Die Kranke bustet und hat Schmerzen in scrobiculo cordis und in beiden Hypochondrien. Es erfolgt Erbrechen selbst nach dem Genusse kleiner Quantiäten Speise. Die Kranke bat keinen Appetit und leidet an Schlaflosigkeit.

30. Oetober. Das Erbrechen hat aufgehört; es habea sich Schlaf und Appetit eingestelt; der Husten danert fort. Der Iterus befindet sich an seinem normalen Orte.

4. November. Der Husten und die Schmerzen in scrobiculo cordis and in beiden Hypochondrien haben bis zn diesem Tage, nach Gebrauch pon Tinct. Yal. aetherea und warmen Wanpenbaddern beinahe aufgehört.

5. November. Es sind nur leichte Schmerzen in der Brust and im Rückea geblieben. Der Jterus befindet sich an seinem normalen Orte.

Bis zam 20. November sind alle krankbaften Erscheinungen verschwunden. Schon seit 14 Tagen hilf die liranke den Krankenwäterinnen bei ibren Arbeiten, wobei das Heben ron Lasten, die für das Alter and die Kräfte dieser Person bedeutend zu nennen waren, keinen Rückfall in ihre lirankheit hervorruft.

In diesen Falle waren zwei Bestreichangen zur vollständigen Heilung hinreichend; gewöhnlich sind mehr nothwendig, aber. nie labe ich mebr als $6 \mathrm{Be}-$ streichungen anwenden mitssen. Ans diesem Falle, sowite aus den anderen, die ich beobachtet habe, habe jch den Schluss gezogen, dass der beste Erfolg bei Beobachlung folgender Bedingungen zu erwarten ist:

1) Es muss möglich sein, den Uterus za repontren.

2) Ehe main zun Anwentung der oben besebriebenen Methode scbreitet, müssen, so weit als möglich, alle anderen Leiden der. Vagina und des Uterus, z. B. Erosionen, Ulcerationen etch, gehoben werden, wenn solche anfangs existiren, es börnten sonst inflammatorische Erscheinungen in der Vagina oder im Uterus eintreten.

3) Es muss nur das Gewölbe der Vagina bestrichen werden, anfangs mit verdünnten Lösungen und schwachen Dosen; bei den späteren Bestreichungen müssen die Stärke der Lösungen and die Grösse der Dosen nach und nach verstärkt werden. Dabei müssen kalte Vaginal-Douchen gebraucht werden, als Mittel, die möglicherweise eintretende Reizung der Vagina und dęs Uterus zu vermindern.

4) In den weisten Fullen braucht die Kranke, nach 2 Bestreichungen, die horizontale Lage nicht nelsr streng einzuhalten. Nach einer $1 \frac{1}{2}$ wöchentlichen Behandlung kann sie gehen und nicht zu schwere Arbeiten verrichten ohne besonderen Schaden.

5) Der Darmkanal puss so viel als möglich rein gebalten werden.

0) Das Intervall zwischen den Bestreichungen darf nicht kleiner sein als 3 Tage, wieder un die keizang der Yagina und des Vterus zu vermeiden. Nach Beendigung der Behandlugg mit Jodlösung mässen die Vaginal-Douchen aus Wasser von $20^{\circ} \mathrm{R}$. einige Zeit fortgesetzt werden.

Nach Beendigung der Cur ist die Vagina weder bemerkbar enger geworden als vorher, noch ist sie dicker als vor derselben. Oft zeigen sich nach Reposition des Iterus sympathische Leiden des Magens, die jedoch leicht und schnell zu 
heben sind. Ich tönnte noch Vieles hinsichtlich der Prognose binzufügen, insoweit sie von den ätiologischen Momenten wenigstens der von mir beobachteten Fälle ablängt, sowie über die anderen bierhergehörigen Fragen.

Ich bin bereit, darüber eine ausfübrlichere Mittheilung zu machen, wenn es dieser Arbeit gelingen sollte, die geneigte Aufmerksamkeit der klinischen Aerzte zu erregen.

5.

Warme Salzbäder bei fieberkranken Kindern.

Von Dr. Carl Schwalbe, Privatdocenten in Zürich.

Bei der Anwendung kalter Bäder zur Herabsetzung der Fiebertemperatur in der Kinderpraxis, hesonders bei Kindern von 1-2 Jahren, hat man oft mit grossen, bisweilen sogar unüberwindlichen Schwierigkeiten zu kämpfen. Ich versuchte daher in einem Falle von katarrhalischer Pneumonie bei einem sebr sebwächlichen, rachitischen, etwas äber $1 \mathrm{Jahr}$ alten Kinde warme Salzbäder $(3-5 \mathrm{pCt}$. Kochsalz) von $30-3 I^{\circ} \mathrm{C}$. zu geben und bin mit den Erfolgen so zufrieden, dass ich nar dringend dazu auffordern kann, dieselben recht bänfig anzuwenden.

Sophie G., 1 Jahr 2 Monate alt, sehr schwächlich, rachitisch, dem Ernährungszustande nach einem schwächlichen Kinde von 6 Monaten gleich, erkrankte am 6. Mai 1871 an acutem Rachen- und Kehlkopflkatarrh. Calomel in abführender Dosis, dann Kali chloric.

8. Mai. Es hat sich katarrhalische Pneumonie ausgebildet, starkes Spielen der Nasenflügel, tiefes Einzieben der unteren Rippen, Husten, katarrhalische Geräusche, hobe Temperatur. Nachm. 3 Uhr T. 39,9.

10. Mai Nachm. 3 Uhr T. 39,9 .

Vollbad mit Salzwasser ( 4 pCt. Kochsalz T. $31,5^{\circ} \mathrm{C}$ ), Dauer $10 \mathrm{Min}$. Kind fühlt sich in dem Bade sehr behaglich, schreit gar nicht.

Nachm. $3 \frac{1}{2}$ Uhr T. 38,1.

NB. Alle Temp. sind von mir im Rectum gemessen. Bei der Messung nach dem Bade, die meistens nach cirea 15 Min. stattand, wurde das Thermometer so lange im Rectum gelassen, bis die Temperatur 5 Min. constant blieb. In der Nacht fünfmal Convulsionen.

11. Mai Abds. 7 Dhr T. 40,0 R. 40 .

Bad ron $32^{\circ}$ C. 12 Min.; T. 38,0 .

Schläft $1 \frac{1}{2}$ Stunden gut, nimmt gern Nahrung (Liebig'sche Suppe).

Abds. 9 Uhr T. 39,2 .

Abds. 10 Uhr T. 39,6.

Bad 10 Min.; T. 38,2 .

12. Nai Morg. 2 Uhr T. 40,0 .

Bad 10 Min.

Archiv f. pathol. Anat, Bd. Ir. Hft. $3 a_{;} 4$. 